\title{
Characterization of the ethanol-inducible alc gene- expression system in Arabidopsis thaliana
}

\author{
Hairul A. Roslan ${ }^{1, \dagger}$, Michael G. Salter ${ }^{1, \pm}$, Chris D. Wood ${ }^{1,5}$, Michael R. H. White ${ }^{1}$, Kevan P. Croft ${ }^{1}$, Frances Robson ${ }^{2}$, \\ George Coupland ${ }^{2}$, John Doonan ${ }^{2}$, Patrick Laufs ${ }^{2}$, A. Brian Tomsett ${ }^{1}$ and Mark X. Caddick ${ }^{1, *}$ \\ ${ }^{1}$ Donnan Laboratories and Life Sciences Building, School of Biological Sciences, University of Liverpool, L69 7ZD, UK, and \\ ${ }^{2}$ John Innes Centre, Norwich Research Park, Colney Lane, Norwich NR4 7UH, UK \\ Received 17 April 2001; revised 6 June 2001; accepted 22 July 2001. \\ *For correspondence (fax +44 151794 3655; email caddick@liverpool.ac.uk). \\ 'Present address: Plant Molecular Biotechnology Laboratory, Faculty of Resource Science and Technology, Universiti Malaysia Sarawak, Jalan Dato' Musa, \\ 94300 Kota Samarahan Sarawak, Malaysia. \\ ${ }^{\ddagger}$ Present address: Department of Biology, Adrian Building, University of Leicester, Leicester LE2 7RH, UK. \\ ${ }^{\S}$ Present address: Department of Physiological Sciences, Medical School, Framlington Place, University of Newcastle, Newcastle-upon-Tyne NE2 4HH, UK.
}

\section{Summary}

Controlled expression of transgenes in plants is key to the characterization of gene function and the regulated manipulation of growth and development. The alc gene-expression system, derived from the filamentous fungus Aspergillus nidulans, has previously been used successfully in both tobacco and potato, and has potential for use in agriculture. Its value to fundamental research is largely dependent on its utility in Arabidopsis thaliana. We have undertaken a detailed function analysis of the alc regulon in $A$. thaliana. By linking the alcA promoter to $\beta$-glucuronidase (GUS), luciferase (LUC) and green fluorescent protein (GFP) genes, we demonstrate that alcR-mediated expression occurs throughout the plant in a highly responsive manner. Induction occurs within one hour and is dose-dependent, with negligible activity in the absence of the exogenous inducer for soil-grown plants. Direct application of ethanol or exposure of whole plants to ethanol vapour are equally effective means of induction. Maximal expression using soil-grown plants occurred after 5 days of induction. In the majority of transgenics, expression is tightly regulated and reversible. We describe optimal strategies for utilizing the alc system in A. thaliana.

Keywords: Arabidopsis thaliana, ethanol, chemically inducible expression, plant-expression system, Aspergillus nidulans.

\section{Introduction}

A key tool in plant molecular biology is the development of effective gene-expression systems. The expression of a transgene can be achieved by using constitutive promoters such as the viral CaMV35S promoter (Odel etal., 1985). However, a constitutive promoter is unsuitable when dealing with genes for which inappropriate expression is either highly deleterious or lethal. Additionally, such expression systems are unsuitable for studies where precise temporal regulation is required, for example, where expression of a gene is desired at a specific stage of plant development, or for analysis of mRNA decay properties. Biotechnological application of a regulated expression system may also be important for crop plants; for instance, the conditional expression of pesticides or herbicide resistance; the induction of synchronous flowering of plants; and the production of a conditional male sterility system. In such cases an effective regulated geneexpression system is required.

The optimal system would employ an inexpensive, nontoxic inducer whose application can be fully controlled, and would lead to a reversibly dose-dependent expression with the potential to achieve high levels of gene expression but with negligible basal activity. To these ends, a number of regulated gene-expression systems have been 